\title{
Incorporation of Line Quantities in the Continuum Description for Multiphase, Multicomponent Bodies with Intersecting Dividing Surfaces
}

\author{
III. Determination of Line Tension and Fluid - Solid Surface Tensions Using Small Sessile Drops
}

\author{
Leonard M. C. Sagis and John C. Slattery ${ }^{1}$ \\ Department of Chemical Engineering, Texas A\&M University, College Station, Texas 77843-3122
}

Received May 24, 1994; accepted May 2, 1995

\begin{abstract}
In this paper we analyze the sessile drop problem, starting from the governing equations for the static problem (part II of this series). We develop a singular perturbation solution, where the perturbation parameter characterizes the strength of the longrange intermolecular forces. We use this solution to develop a new method to determine line tension and solid - fluid surface tensions, using small sessile drops. $\odot 1995$ Academic Press, Inc.

Key Words: common line; three-phase line of contact; line tension; sessile drop; solid - fluid surface tension.
\end{abstract}

\section{INTRODUCTION}

In the first two papers of this series $(1,2)$, we derived the governing equations and proposed a description of material behavior for the common lines of multiphase, multicomponent systems, taking into account the contribution of line excess quantities (line mass density, line velocity, . . .).

There is little experimental evidence that line excess quantities have a significant influence on the system as a whole. Line tension seems to be the exception. De Feijter and Vrij (3), Platikanov et al. $(4,5)$, and Kralchevsky and Ivanov $(6,7)$ studied the effects of line tension in thin soap films. Langmuir (8), Harkins (9), and Torza and Mason (10) examined the effects of line tension on the configuration of fluid lenses. Good and Koo (11), Starov and Churaev (12), Gaydos and Neumann (13), Wallace and Schurch (14), Yekta-Fard and Ponter (15), Li et al. (16), and Drelich et al. (17) considered the effects of line tension on the macroscopic contact angle of a sessile drop. Grets (18), Navascues and Mederos (19), and Lazaridis (20) discussed the effects of line tension in heterogeneous nucleation. Churaev and Starov (21) studied the effects of line tension in the liquid layer between two emulsion droplets.

In this paper we will study the sessile drop, starting from the governing equations for the static problem we derived in Section 6 of part II. We seek the relationship between the

\footnotetext{
${ }^{1}$ To whom correspondence should be addressed.
}

magnitude of the macroscopic contact angle and the diameter of the sessile drop.

The configuration of the sessile drop is given in Fig. 1. Within the immediate neighborhood of the common line, the meniscus is very thin, and the liquid/vapor interface forms an angle $\Theta_{0}$ with the solid surface. We refer to this angle as the intrinsic contact angle, and we refer to this part of the sessile drop as the inner region, in which long-range intermolecular forces are important. Sufficiently far away from the common line, the liquid/vapor interface forms an angle $\Theta^{\text {(stat) }}$ with the solid surface. We refer to $\Theta^{\text {(stat) }}$ as the static contact angle or macroscopic contact angle. It is the angle an experimentalist would observe at perhaps $10 \times$ magnification. We refer to the region outside the immediate neighborhood of the common line as the outer region, in which long-range intermolecular forces can be neglected.

Bashforth and Adams (22) were the first to report accurate numerical solutions for the outer region by solving the jump momentum balance (Laplace equation) for the liquid/vapor interface. Their work was extended by Blaisdell (23-25), Staicopolus (26-28), Padday (29), Padday and Pitt (30), and Hartland and Hartley (31).

Dzyaloshinskiǐ et al. (32), Padday and Uffindell (33), and Hough and White (34) derived expressions for the static contact angle of a sessile drop, starting from the tangential component of the momentum balance at the common line [Young's equation (35)], and using expressions for the surface tensions in terms of long-range intermolecular forces. Israelachvili (36) derived expressions for the static contact angle using a similar approach, but starting from the YoungDupré equation.

Miller and Ruckenstein (37) and Jameson and del Cerro (38) studied the inner region. They derived expressions for the static contact angle, assuming a wedge-shaped profile for the liquid film in this region.

Saville (39) calculated values for the static contact angle using molecular dynamics. Benner et al. (40) calculated density and stress profiles in the inner region, using gradient theory. 

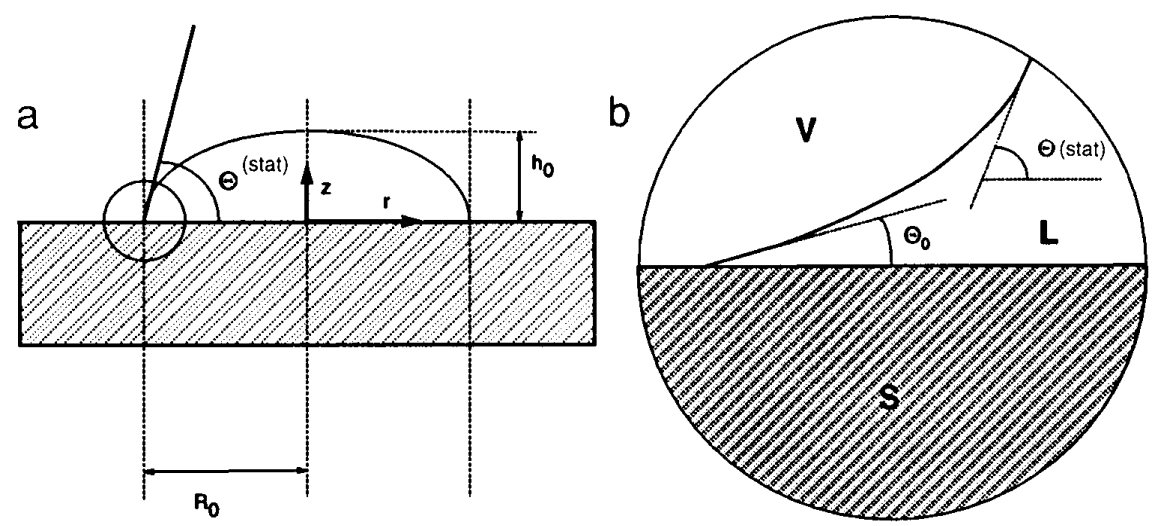

FIG. 1. (a) The configuration of the sessile drop. (b) Close up of the configuration in the immediate neighborhood of the common line.

Wayner $(41,42)$ calculated the configuration of the liq$\mathrm{uid} /$ vapor interface in the inner region and values for $\Theta^{\text {(stat) }}$, starting from the jump momentum balance.

The work presented here is an extension of the work presented by Li and Slattery (43, Section 3.2.7). They calculated the configuration of the liquid/vapor interface using a singular perturbation scheme, in which the perturbation parameter characterizes the strength of the long-range molecular forces. They determined $\Theta^{\text {(stat) }}$ for several $n$-alkanes on PTFE, and they compared their results with values measured for these systems by Fox and Zisman (44). They found that there was reasonable agreement between theory and experiment for low values of the carbon number ( $n$ $=5-7)$. For higher carbon numbers their results differed significantly from the experimental values. Several explanations were suggested for this discrepancy:

1. They account only for the contributions from Londonvan der Waals forces, neglecting any contributions from electrostatic double-layer, steric, and structural forces.

2. They approximated the contribution of the Londonvan der Waals forces by using an expression for a system consisting of a thin liquid film of uniform thickness, bounded by two semi-infinite phases.

3. They neglected any effects of electromagnetic retardation on the London-van der Waals forces.

4. They assumed that the intrinsic contact angle $\Theta_{0}=0$.

To test the validity of assumption 2 , they replaced the expression for the London-van der Waals forces by the more realistic expression for a wedge-shaped film $(37,38)$. They found that the effect of this change was less than $2 \%$.

In this paper we will first try to improve the approach suggested by Li and Slattery (43, Section 3.2.7) by addressing each of the remaining assumptions they thought were responsible for the unsatisfactory agreement between theory and experiment. As they suggested, we will develop a singular perturbation solution, where the perturbation parameter characterizes the strength of the long-range intermolecular forces. The solution separates into an outer region in which long-range intermolecular forces are negligible and an inner region where these effects are dominant. We will discuss the validity of assumption 1 in Section 2. In this section we will also discuss the incorporation of electromagnetic retardation effects in the description of the Londonvan der Waals forces. The validity of assumptions 3 and 4 will be discussed in Section 4.

After identifying the cause of the discrepancy between theory and experiment, we will use the theory to investigate the dependance of the macroscopic contact angle on the size of the sessile drop. Several authors have reported a dependance of the static contact angle on the size of the sessile drop. Good and Koo (11) reported a decrease of the static contact angle with decreasing drop size. Gaydos and Neumann (13) reported an increase in the static contact angle with decreasing drop size. In both studies the authors attribute the observed effects to line tension, a negative line tension in the case of Good and Koo (11), and a positive line tension in the case of Gaydos and Neumann (13).

\section{INTERMOLECULAR FORCES}

In the immediate neighborhood of the common line, the liquid film is very thin, and the effects of intermolecular forces are very important. In this section we will discuss the intermolecular forces that contribute to the total force at the liquid/vapor interface of this thin film.

Intermolecular forces are usually divided into two classes: long-range and short-range forces. Long-range forces can have effects at interfacial separation distances of up to 1000 $\AA$; short-range forces rarely have any effects at separation distances beyond $10 \AA$. The three most important long-range forces are the London-van de Waals or dispersion forces, the electrostatic double-layer forces, and the steric forces. The dominant short-range forces are the structural forces (solvation or hydration forces).

Electrostatic double-layer forces arise, when the interfaces are charged, as the result of adsorption of ions from solution or dissociation of surface groups. We will confine ourselves 
to nonpolar, nondissociative liquids on a nonpolar, nondissociative surface.

Steric forces occur when the interfaces are covered with polymers or surfactants with a long chain segment. When two interfaces approach one another, the polymer or surfactant chains overlap, leading to a repulsive force between the interfaces. We assume here that no polymers or surfactants are present, and steric forces do not contribute to the total intermolecular force.

Structural forces can arise when the two interfaces are very close together, inducing liquid molecules to order into layers within a highly restricted space. When both surfaces are rigid, these forces have a rapidly decaying, oscillatory character. They vary between attraction and repulsion with a periodicity approximately equal to the diameter of the liquid molecules (45-49). For fluid-fluid interfaces, the oscillations are smoothed. The resulting force is a smoothly varying attractive force, which is well described using the description of the van der Waals forces (46). Since we have one fluid-fluid interface, we will not have to include the effects of structural forces, and assumption 1 can be eliminated as the cause for the discrepancy between theory and experiment reported by Li and Slattery (43, Section 3.2.7).

This leaves us with only one type of intermolecular forces to consider: the London-van der Waals forces. These forces are usually monotonically attractive, describing the forces arising from dipole-dipole (orientation) interactions, dipoleinduced dipole (induction) interactions and, induced dipoleinduced dipole (dispersion) interactions. Since we will confine ourselves to nonpolar substances, we will only have to consider the contributions of the dispersion interactions.

\section{The London-van der Waals Forces}

Let us consider a point $\mathbf{z}_{(i)}$ in phase $i(i=\mathrm{V}, \mathrm{L}, \mathrm{S})$ and a point $\mathbf{z}_{(j)}$ in phase $j$. Here $\mathrm{V}$ denotes the vapor phase, $\mathrm{L}$ the Liquid phase, and $S$ the solid phase. The force per unit mass of $i$ per unit mass of $j$ at point $\mathbf{z}_{(i)}$, resulting from intermolecular interactions with point $\mathbf{z}_{(j)}$, is denoted by $\mathbf{b}_{(i j)}$. We will assume that this force can be represented as

$$
\mathbf{b}_{(i j)}=-\nabla \phi_{(i j)},
$$

where $\phi_{(i j)}$ denotes the pair potential per unit mass of $i$ per unit mass of $j$ at $\mathbf{z}_{(i)}$. We will assume that

$$
\phi_{(i j)}=-\frac{C_{(i j)}}{r_{(i j)}^{6}},
$$

where $r_{(i j)}$ denotes the distance between the points $\mathbf{z}_{(i)}$ and $\mathbf{z}_{(j)}$. This particular form of the pair potential is referred to as the London $(50$, p. 30$)$ or Sutherland potential (51, p. 531 ). It takes into account induced dipole-induced dipole interactions, but it ignores contributions from higher order multipoles (for example induced quadrupole-induced quad- rupole). Note that $\phi_{(i j)}$ decays as $r_{(i j)}^{-6}$, and we speak of unretarded London-van der Waals forces. Casimir and Polder (52) found that [2] is valid only for small values of $r_{(i j)}$. For larger values, they found the potential decays more rapidly, as a result of electromagnetic retardation effects. For $r_{(i j)}>1000 \AA$, it decays as $r_{(i j)}^{-7}$, and they denote this dependance as the fully retarded limit.

There are two principal methods for calculating the total force at a point $\mathbf{z}_{(i)}$ resulting from the intermolecular interactions with all of the surrounding material. Hamaker (53) assumed additivity of the pairwise interactions in calculating the interaction potential of a sphere attributable to the presence of another sphere in a vacuum. Applying this approach to our system, we find that

$$
\begin{aligned}
\mathbf{b}_{(i)} & =-\sum_{j=\mathrm{V}, \mathrm{L}, \mathrm{S}} \int_{R_{(j)}} \rho_{(j)} \nabla \phi_{(i j)} d V \\
& =-\nabla\left[\sum_{j=\mathrm{V}, \mathrm{L}, \mathrm{S}} \int_{R_{(j)}} \rho_{(j)} \phi_{(i j)} d V\right] \\
& =-\nabla \Phi_{(i)}^{\mathrm{LvW}},
\end{aligned}
$$

where $\mathbf{b}_{(i)}$ is the force per unit mass of $i$ at point $\mathbf{z}_{(i)}$, $\Phi_{(i)}^{\mathrm{LvW}}$ is the potential energy per unit mass of $i$ attributable to London-van der Waals forces, $\rho_{(j)}$ is the density of phase $j$, and $R_{(j)}$ is the domain of phase $j$.

For condensed media, the assumption of additivity of pairwise interactions is incorrect, because it ignores the influence of the material in the immediate neighborhood of the two points on the interactions between them. We must recognize that the polarization of a molecule can be influenced by the material in its immediate neighborhood.

In his calculation of the interactions between two bodies separated by a vacuum gap of uniform thickness, Lifshitz (54) avoids using pairwise additivity by treating the bodies as a continuum and by treating the interactions between these two continua as though they occur through interactions of their fluctuating electromagnetic fields. In this approach, multibody and retardation effects are automatically included. Dzyaloshinskii et al. (55) generalized the Lifshitz theory for the case in which the gap between the two media is not a vacuum.

Lifshitz theory gives significantly better results than the approach used by Hamaker (53). Since the computations are complex, Israelachvili (47, p. 180) suggested that the approach of Hamaker could be used, so long as the Hamaker constant is calculated using Lifshitz theory.

Consider a system consisting of a thin liquid film of uniform thickness $h^{\prime}$ between a semi-infinite vapor phase and a semi-infinite solid phase. Using the approach suggested by Hamaker (53), we find for this system that at the liquid/ vapor interface $(56,57)$

$$
\rho_{(\mathrm{L})} \Phi_{(\mathrm{L})}^{\mathrm{LvW}}-\rho_{(\mathrm{V})} \Phi_{(\mathrm{V})}^{\mathrm{LvW}}=\Phi_{\infty}^{(\mathrm{LV})}+\frac{B}{h^{\prime m}},
$$


where $\Phi_{\infty}^{(\mathrm{LV})}$ denotes the interaction potential per unit volume of a semi-infinite liquid film in the limit as the interface is approached,

$$
B=\frac{1}{6 \pi} A,
$$

and

$$
m=3 .
$$

We refer to $A$ as the Hamaker constant. Using Lifshitz theory, Vassilieff and Ivanov (58-60; see also 61) found

$$
\begin{aligned}
A=A_{n=0}+A^{(\mathrm{LL})}+A^{(\mathrm{VS})} & \\
& -A^{(\mathrm{VL})}-A^{(\mathrm{LS})}+\delta A^{(\mathrm{LVS})} .
\end{aligned}
$$

Here $A_{n=0}$ is the zero-frequency term; for nonpolar compounds it is zero. The term $\delta A^{(\text {LVS })}$ is a correction for nonadditivity; we will neglect the contribution of this term. The $A^{(i j)}$ terms are given by

$$
\begin{gathered}
A^{(i j)}=\frac{3 \hbar}{4 \pi} \frac{a_{(i)} a_{(j)}}{b_{(i)}+b_{(j)}}\left[1-\left(\frac{D_{(i j)}}{D_{(i j)}+1}\right)^{3}\right] \\
D_{(i j)}=\frac{\left(n_{(\mathrm{L})}+1\right) h^{\prime}}{c\left(b_{(i)}+b_{(j)}\right)},
\end{gathered}
$$

where $c$ denotes the speed of light in vacuum, and $n_{(\mathrm{L})}$ denotes the refractive index of the liquid phase; $a_{(j)}$ and $b_{(j)}$ denote the coefficients in the empirical relation for the dielectric permeability $\varepsilon_{(j)}(i \omega)$, suggested by Krupp (62):

$$
\varepsilon_{(j)}(i \omega)=\frac{1+a_{(j)} \exp \left(-b_{(j)} \omega\right)}{1-a_{(j)} \exp \left(-b_{(j)} \omega\right)} .
$$

For nonpolar materials $a_{(j)}$ can be related to the refractive index of that material through

$$
a_{(j)}=\frac{n_{(j)}^{2}-1}{n_{(j)}^{2}+1} .
$$

The term in square brackets in [8] denotes the correction factor for retardation effects.

In order to use [8], we need to find values for $a_{(j)}$ and $b_{(j)}$. In this paper we will confine ourselves to systems where the liquid is an $n$-alkane and the solid is Teflon. The third phase of the system is a vapor. Since a vapor has a very

\begin{tabular}{|c|c|c|c|c|c|}
\hline Compound & $n_{(\mathrm{L})}$ & $\begin{array}{c}A_{0}^{(\mathrm{LL})} \\
{\left[10^{-20} \mathrm{~J}\right]^{a}}\end{array}$ & $\begin{array}{c}A_{0}^{(\mathrm{LS})} \\
{\left[10^{-20} \mathrm{~J}\right]^{a}}\end{array}$ & $a_{(\mathrm{L})}$ & $\begin{array}{c}b_{(\mathrm{L})} \\
{\left[10^{-17} \mathrm{~s}\right]}\end{array}$ \\
\hline Dodecane & 1.4216 & 5.03 & 4.35 & 0.338 & 7.162 \\
\hline Tetradecane & 1.4290 & 5.09 & 4.38 & 0.3425 & 7.190 \\
\hline Hexadecane & 1.4345 & 5.22 & 4.43 & 0.346 & 6.967 \\
\hline
\end{tabular}
low density, compared to a liquid or a solid, we will assume that $A^{(\mathrm{VS})} \approx A^{(\mathrm{VL})} \approx \delta A^{(\mathrm{LVS})} \approx 0$. Hence we only must concern ourselves with the evaluation of the coefficients for the $n$-alkanes and Teflon. For Teflon, Vassilieff and Ivanov
TABLE 1

Physical Constants U sed in the Calculation of the Hamaker Constant for Dodecane, Tetradecane, and Hexadecane

${ }^{a}$ Values for the unretarded Hamaker constants were taken from Hough and White (34).

(59) calculated that $a_{(\mathrm{S})}=0.317$ and $b_{(\mathrm{S})}=1.3195 \times 10^{-16}$ s. In Table 1 we give the values for $a_{(\mathrm{L})}$ and $b_{(\mathrm{L})}$ for the three $n$-alkanes we will study in this paper. The values for $a_{(\mathrm{L})}$ were obtained using Eq. [11]. The values for $b_{(\mathrm{L})}$ were obtained by taking the unretarded limit for both $A^{(\mathrm{LL})}$ and $A^{(\mathrm{LS})}$ (by setting $D_{(i j)}$ to zero in Eq. [8]), and equating the results to values for this limit obtained by Hough and White (34). The expression we used is given by

$$
A_{0}^{(\mathrm{LL})}-A_{0}^{(\mathrm{LS})}=\frac{3 \hbar}{4 \pi}\left[\frac{a_{(\mathrm{L})}^{2}}{2 b_{(\mathrm{L})}}-\frac{a_{(\mathrm{L})} a_{(\mathrm{S})}}{b_{(\mathrm{L})}+b_{(\mathrm{S})}}\right] .
$$

For $A_{0}^{(\mathrm{LL})}$ and $A_{0}^{(\mathrm{LS})}$ we substituted the values calculated by Vassilieff and Ivanov (59); finally, for $a_{(\mathrm{L})}$ we substituted the value given by Eq. [11], leaving us with a single equation, in which $b_{(\mathrm{L})}$ is the only unknown variable.

\section{PROBLEM STATEMENT}

We will make the following assumptions:

(i) The configuration of the sessile drop is depicted in Fig. 1. The solid is assumed to be rigid; its surface is smooth and planar. The common line is assumed to be circular. Within the immediate neighborhood of the common line, the meniscus is very thin. Here the liquid/vapor interface forms an angle $\Theta_{0}$ with the solid surface. Sufficiently far away from the common line, the liquid/vapor interface forms an angle $\Theta^{\text {(stat) }}$ with the solid surface.

(ii) The system is at equilibrium. All physical properties are constants.

(iii) We will use a cylindrical coordinate system $(r, \theta$, $z$ ). The $z=0$ plane is assumed to be coincident with the solid surface, and the $z$-axis is assumed to be perpendicular to the solid surface. The origin is assumed to be at the center of the sessile drop.

(iv) We will assume that the surface mass density $\rho^{(\sigma)}$ and the line mass density $\rho^{(\mathrm{cl})}$ are negligibly small.

(v) The liquid and vapor phase are incompressible.

(vi) We will assume that all external and mutual forces can be represented as the gradient of a potential energy per unit mass. 
(vii) We will account for both gravity and London-van der Waals forces within the immediate neighborhood of the common line. As discussed in Section 2, we will neglect any contributions from electrostatic double-layer forces, steric forces, and structural forces. We will approximate the contribution of the London-van der Waals forces by using the expression derived for a system consisting of a liquid film of uniform thickness $h^{\prime}$, between a semi-infinite vapor and semi-infinite solid phase $(56,57)$. Using [4], we find at the liquid/vapor interface that

$$
\begin{aligned}
\rho_{(\mathrm{L})} \Phi_{(\mathrm{L})}-\rho_{(\mathrm{V})} & \Phi_{(\mathrm{V})} \\
& =\Phi_{\infty}^{(\mathrm{LV})}+\frac{B}{h^{\prime m}}+\left(\rho_{(\mathrm{L})}-\rho_{(\mathrm{V})}\right) g h^{\prime} .
\end{aligned}
$$

(viii) We will account for retardation effects in the description of the London-van der Waals forces ( $B$ and $m$ are given by [5] and [6]; $A$ is given by [7]).

(ix) We will neglect any contributions from the vapor phase to the London-van der Waals forces, which implies that the expression for $A$ reduces to

$$
A=A^{(\mathrm{LL})}-A^{(\mathrm{LS})},
$$

where $A^{(\mathrm{LL})}$ and $A^{(\mathrm{LS})}$ are calculated using [8].

(x) Israelachvili (36) suggests that the distance between two interfaces cannot go to zero due to the finite size of the constituent atoms and that a finite phase separation distance $d$ must be recognized. He recommends that $d$ be viewed as the mean distance between the centers of the individual atoms and estimated as

$$
d=0.91649\left[M /\left(\rho n_{\mathrm{a}} N\right)\right]^{1 / 3},
$$

in which $M$ is the molecular weight, $n_{\mathrm{a}}$ is the number of atoms per molecule, and $N$ is Avogadro's constant (6.023 $\times 10^{23} \mathrm{~mol}^{-1}$ ). In arriving at this result, the atoms have been assumed to be in a close packing arrangement. For a molecule consisting of a repeating unit, such as an $n$-alkane, we suggest a simple picture in which the molecules are arranged in such a manner that the repeating units $\left(-\mathrm{CH}_{2}-\right.$ groups) are in a close packing arrangement (43, Section 3.2.7):

$$
d=0.91649\left[M /\left(\rho n_{\mathrm{u}} N\right)\right]^{1 / 3} .
$$

Here $n_{\mathrm{u}}$ is the number of repeating units in the molecule. For the minimum separation between the liquid/vapor and the liquid/solid interface, we suggest (43, Section 3.2.7) that

$$
d=\left(d_{(\mathrm{L})}+d_{(\mathrm{S})}\right) / 2,
$$

where $d_{(\mathrm{L})}$ and $d_{(\mathrm{S})}$ are calculated using Eq. [16]. (xi) We follow Wayner $(41,42)$ in recognizing that the minimum film is a monolayer. We will estimate the thickness of this monolayer as $d$. This might now be thought of as the distance between the centers of the last layer of solid atoms and the centers of the first layer of liquid atoms or repeating units. We will measure the film thickness $h$ from the centers of the first layer of liquid atoms or repeating units, replacing [13] by

$$
\begin{aligned}
\rho_{(\mathrm{L})} \Phi_{(\mathrm{L})}- & \rho_{(\mathrm{V})} \Phi_{(\mathrm{V})} \\
& =\Phi_{\infty}^{(\mathrm{LV})}+\frac{B}{(h+d)^{m}}+\left(\rho_{(\mathrm{L})}-\rho_{(\mathrm{V})}\right) g h .
\end{aligned}
$$

In this simplistic model, the common line can be visualized as running through the centers of the liquid atoms or repeating units on the leading edge of the monolayer.

\section{ANALYSIS}

In the coordinate system described in assumption (iii), the liquid-vapor interface can be represented as

$$
z=h(r)
$$

For the sessile drop problem the governing equations are the differential momentum balance for the liquid and the vapor phases, the jump momentum balance for the liquid/ vapor interface, and the momentum balance at the common line (2).

With assumptions (v) and (vi), the differential momentum balance for the liquid and the vapor phases reduces to

$$
\nabla P_{(j)}+\rho_{(j)} \nabla \Phi_{(j)}=0 \quad \text { for } j=\mathrm{L}, \mathrm{V}
$$

in which $P_{(j)}$ denotes the pressure in phase $j$. With assumptions (ii) and (iv), the jump momentum balance for the liquid/vapor interface reduces to

$$
P_{(\mathrm{V})}-P_{(\mathrm{L})}=2 \gamma_{(\mathrm{LV})} H,
$$

where the mean curvature is of the form (43, p. 269)

$$
\begin{aligned}
H=\frac{1}{2 r}\left[r \frac{d^{2} h}{d r^{2}}+\frac{d h}{d r}+\left(\frac{d h}{d r}\right)^{3}\right] & \\
\times & {\left[1+\left(\frac{d h}{d r}\right)^{2}\right]^{-3 / 2} . }
\end{aligned}
$$

Finally, we can simplify the momentum balance at the common line, using assumptions (ii) and (iv). The tangential component of that equation (with respect to the solid surface) is (2) 


$$
\gamma_{(\mathrm{LS})}-\gamma_{(\mathrm{SV})}+\gamma_{(\mathrm{LV})} \cos \Theta_{0}+\sigma \kappa_{\nu}^{(\mathrm{L}, \mathrm{S})}=0,
$$

where $\kappa_{\nu}^{(\mathrm{L}, \mathrm{S})}$ denotes the geodesic curvature of the common line with respect to the liquid/solid interface. Equation [23] is sometimes referred to as the generalized Young equation.

Integrating [20] for both the liquid and the vapor phase, we find

$$
P_{(j)}+\rho_{(j)} \Phi_{(j)}=C_{(j)} \quad \text { for } j=\mathrm{L}, \mathrm{V},
$$

where $C_{(j)}$ is an integration constant. Substituting this result into [21] and using assumptions (viii) and (xi) we find

$$
G+\frac{A}{6 \pi(h+d)^{3}}+\left(\rho_{(\mathrm{L})}-\rho_{(\mathrm{V})}\right) g h=2 \gamma_{(\mathrm{LV})} H .
$$

Here we have defined

$$
G \equiv C_{(\mathrm{V})}-C_{(\mathrm{L})}+\Phi_{\infty}^{(\mathrm{LV})} .
$$

We will find it convenient to introduce as dimensionless variables

$$
\begin{aligned}
h^{*} & \equiv \frac{h}{R_{0}} \quad h_{0}^{*} \equiv \frac{h_{0}}{R_{0}} \quad r^{*} \equiv \frac{r}{R_{0}} \\
d^{*} & \equiv \frac{d}{R_{0}} \quad G^{*} \equiv \frac{G R_{0}}{\gamma_{(\mathrm{LV})}} \quad A^{*} \equiv \frac{A}{6 \pi \gamma_{(\mathrm{LV})} R_{0}^{2}} \\
H^{*} & \equiv H R_{0},
\end{aligned}
$$

in which $R_{0}$ denotes the radius at the base of the sessile drop. This permits us to express [25] as

$$
G^{*}+\frac{A^{*}}{\left(h^{*}+d^{*}\right)^{3}}+N_{\mathrm{B} 0} h^{*}=2 H^{*},
$$

where

$$
N_{\mathrm{B} 0} \equiv \frac{\left(\rho_{(\mathrm{L})}-\rho_{(\mathrm{V})}\right) g R_{0}^{2}}{\gamma_{(\mathrm{LV})}}
$$

is the Bond number.

Let us denote the unretarded limit for $A^{*}$ by $A_{0}^{*}$. Since $\left|A_{0}^{*}\right| \ll 1$, our objective is to develop a solution for [28] that is correct in the limit $\left|A_{0}^{*}\right| \rightarrow 0$ or a perturbation solution that is correct to the zeroth order in $\left|A_{0}^{*}\right|$.

Outside the immediate neighborhood of the common line, the liquid film is no longer very thin. In this region, Eq. [28] reduces in the limit $\left|A_{0}^{*}\right| \rightarrow 0$ to

$$
G^{*}+N_{\mathrm{B} 0} h^{*}=2 H^{*}
$$

which must be solved consistent with

$$
\begin{gathered}
\text { at } r^{*}=0, \quad h^{*}=h_{0}^{*} \\
\text { at } r^{*}=0, \quad \frac{d h^{*}}{d r^{*}}=0 \\
\text { as } r^{*} \rightarrow 1, \quad \frac{d h^{*}}{d r^{*}} \rightarrow-\tan \Theta^{(\mathrm{stat})} .
\end{gathered}
$$

We will refer to [30] through [33] as the outer problem.

Within the immediate neighborhood of the common line, the liquid film is very thin, and the effects of the Londonvan der Waals forces are dominant. Let us define

$$
\begin{aligned}
& h^{* *} \equiv \frac{h}{R_{0}}\left|A_{0}^{*}\right|^{-1 / 2} \\
& r^{* *} \equiv \frac{r}{R_{0}}\left|A_{0}^{*}\right|^{-1 / 2} \quad s^{* *} \equiv\left|A_{0}^{*}\right|^{-1 / 2}-r^{* *} \\
& d^{* *} \equiv \frac{d}{R_{0}}\left|A_{0}^{*}\right|^{-1 / 2} \quad G^{* *} \equiv \frac{G R_{0}}{\gamma_{(\mathrm{LV})}}\left|A_{0}^{*}\right|^{1 / 2}
\end{aligned}
$$

In terms of these expanded variables, Eq. [28] reduces in the limit $\left|A_{0}^{*}\right| \rightarrow 0$ to

$$
G^{* *}+\frac{A^{*}}{\left|A_{0}^{*}\right|} \frac{1}{\left(h^{* *}+d^{* *}\right)^{3}}=2 H^{* *},
$$

where $H^{* *}$ is given by

$$
\begin{aligned}
& H^{* *}=\frac{\left|A_{0}^{*}\right|^{1 / 2}}{2} {\left[\left|A_{0}^{*}\right|^{-1 / 2} \frac{d^{2} h^{* *}}{d s^{*} *^{2}}-\frac{d h^{* *}}{d s^{* *}}\right.} \\
&\left.-\left(\frac{d h^{* *}}{d s^{* *}}\right)^{3}\right]\left[1+\left(\frac{d h^{* *}}{d s^{* *}}\right)^{2}\right]^{-3 / 2}
\end{aligned}
$$

Equation [35] must be solved consistent with the boundary conditions

$$
\begin{gathered}
\text { at } s * *=0, \quad h * *=0 \\
\text { at } s^{* *}=0, \quad \frac{d h^{* *}}{d s^{* *}}=\tan \Theta_{0}
\end{gathered}
$$

with $\Theta_{0}$ specified by [23]. We will refer to [35] through [38] as the inner problem.

The inner and outer solutions must match in some intermediate region 
as $\left|A_{0}^{*}\right| \rightarrow 0$ and $s^{* *} \rightarrow \infty$,

$$
\frac{d h^{* *}}{d s^{* *}} \rightarrow-\frac{d h^{*}}{d r^{*}} \rightarrow \tan \Theta^{(\mathrm{stat})} .
$$

Using the definition of $G^{* *}$, we can rewrite [35] as

$$
G^{*}\left|A_{0}^{*}\right|^{1 / 2}+\frac{A^{*}}{\left|A_{0}^{*}\right|} \frac{1}{\left(h^{* *}+d^{* *}\right)^{3}}=2 H^{* *} .
$$

Here $G^{*}$ is the shape factor determined by the outer problem. The term $G^{*}\left|A_{0}^{*}\right|^{1 / 2}$ will disappear only if $G^{*}$ is sufficiently small. If $G^{*}$ is sufficiently large, the inner and outer problem will be coupled, and

$$
\Theta^{\text {(stat) }}=\Theta^{\text {(stat) }}\left(d^{* *}, \Theta_{0}, G^{*}\left|A_{0}^{*}\right|^{1 / 2}\right),
$$

which implies that $\Theta^{\text {(stat) }}$ depends on the macroscopic geometry of the sessile drop.

Li and Slattery (43, Section 3.2.7) suggested this as an alternative explanation for the observations made by Good and Koo (11) and Gaydos and Neumann (13). To test this hypothesis, we constructed a solution scheme that allows for the simultaneous solution of the inner and outer problem. First we set $G^{*}=0$ in Eq. [40] and solved for the initial configuration of the liquid/vapor interface and an initial value of $\Theta^{\text {(stat })}$, consistent with [37] and [38]. We used this initial value for $\Theta^{\text {(stat) }}$ to solve for the configuration of the liquid/vapor interface in the outer region, by solving [30] consistent with [31] through [33]. This gave us a new value for $G^{*}$. We substituted this value in [40], and again solved for the configuration of the liquid/vapor interface and a new value for $\Theta^{\text {(stat) }}$. This new value for $\Theta^{\text {(stat) }}$ was used to calculate a new value for $G^{*}$ in the outer problem. This process was repeated until convergence was achieved in both $G^{*}$ and $\Theta^{\text {(stat) }}$.

\section{RESULTS AND DISCUSSION}

In Section 1 we listed four possible reasons for the discrepancy between theory and experimental results in the work done by Li and Slattery (43, Section 3.2.7). In their work, Li and Slattery (43, Section 3.2.7) replaced the expression for the London-van der Waals (Eq. [4]) by the more realistic expression for a wedge-shaped film. They found that the change in their results was less than $2 \%$. This implies that assumption 2 is not responsible for the discrepancy. In Section 2 we discussed the validity of assumption 1 and discarded it as being responsible for the unsatisfactory agreement with experimental results. This leaves us with only assumptions 3 and 4 as the possible explanation for the poor agreement between calculated and measured values for $\Theta^{\text {(stat) }}$.

To check the validity of assumption 3 we determined the
TABLE 2

\section{Physical Constants U sed in the Calculation of the Drop Profile} for Dodecane, Tetradecane, and Hexadecane

\begin{tabular}{lccc}
\hline Compound & $d_{(\mathrm{L})}[\AA]$ & $\gamma\left[10^{-3} \mathrm{~N} / \mathrm{m}\right]^{a}$ & $\rho_{(\mathrm{L})}\left[\mathrm{kg} / \mathrm{m}^{3}\right]$ \\
\hline Dodecane & 2.894 & 25.4 & 748.7 \\
Tetradecane & 2.874 & 26.7 & 762.8 \\
Hexadecane & 2.860 & 27.6 & 773.3 \\
\hline
\end{tabular}

Note. For PTFE we used $d_{(\mathrm{S})}=3.107 \AA$.

${ }^{a}$ Values for the surface tensions were taken from Fox and Zisman (44).

inner and outer solution for three different systems (dodecane, tetradecane, and hexadecane), setting $\Theta_{0}=0$ and using [7] through [9] to calculate the Hamaker constant. In Table 2 we list the numerical values we used for the physical constants of the respective systems. In all three cases we saw no improvement of the results, compared to the results found by Li and Slattery (43, Section 3.2.7). The theory still consistently underestimates the value for $\Theta^{\text {(stat) }}$ (up to $50 \%$ in the case of hexadecane). This leads us to conclude that neglecting the effects of electromagnetic retardation on the London-van der Waals forces is not responsible for the discrepancy between theory and experimental results.

For the system tetradecane on PTFE, we made several calculations, again setting $\Theta_{0}=0$ and changing the value of the radius of the sessile drop between values of $1 \times 10^{-3}$ and $4 \times 10^{-3} \mathrm{~m}$ (the approximate range covered by Gaydos and Neumann (13) in their experiments). For this range of radii, we did not see any dependance of the inner solution on the outer solution. In all cases the inner and outer problem decoupled, hence giving us a single value for $\Theta^{\text {(stat) }}$, which was independent of the radius of the sessile drop. For the range of radii we covered we found that $G^{*}<0$ and $G^{*}$ $\sim 0$ (1), which means that the term $G^{*}\left|A_{0}^{*}\right|^{1 / 2}$ in Eq. [40] vanishes with respect to the remaining terms. We conclude that Li and Slattery's (43, Section 3.2.7) explanation for the dependance of the static contact angle on the radius of the sessile drop cannot explain this dependance for the range of radii covered by the experimental data. For this range, [41] reduces to

$$
\Theta^{(\text {stat })}=\Theta^{(\text {stat })}\left(d^{* *}, \Theta_{0}\right) .
$$

These observations lead us to the conclusion that the assumption that $\Theta_{0}=0$ is responsible both for the discrepancy between theory and experimental results and for the inability of the theory to predict a dependance of $\Theta^{\text {(stat) }}$ on the radius of the sessile drop. Instead of assuming that $\Theta_{0}=0$, we should actually use [23] to calculate $\Theta_{0}$. For a sessile drop with a circular common line, this equation reduces to

$$
\gamma_{(\mathrm{LS})}-\gamma_{(\mathrm{SV})}+\gamma_{(\mathrm{LV})} \cos \Theta_{0}+\frac{\sigma}{R_{0}}=0 .
$$



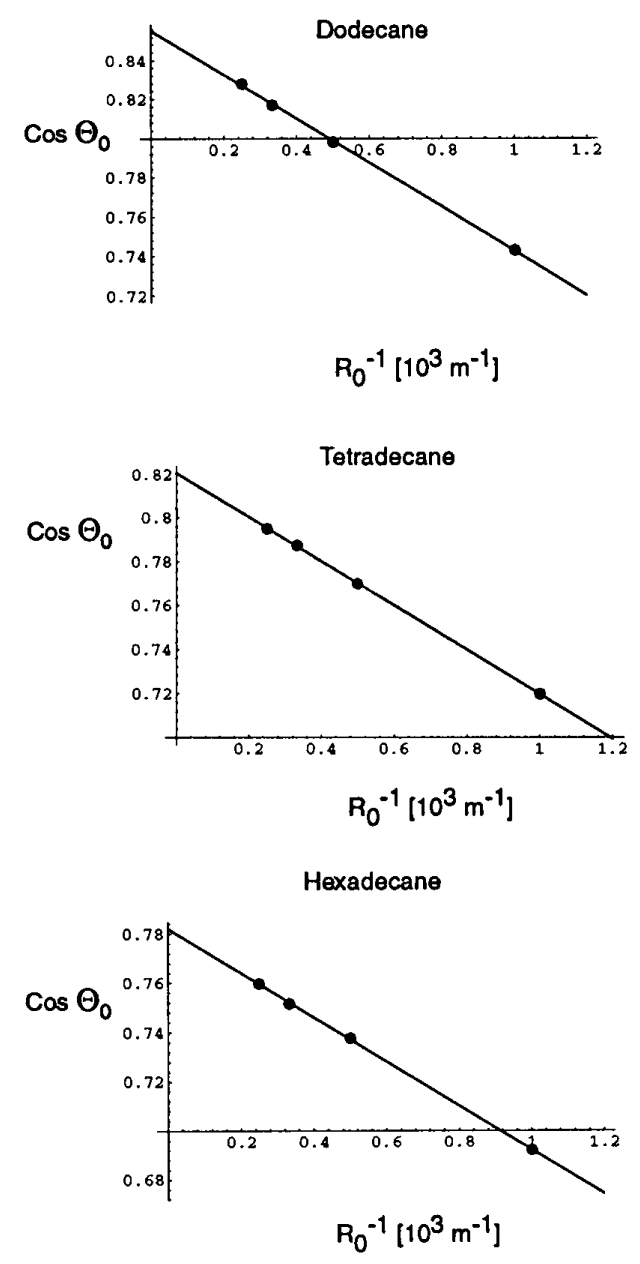

FIG. 2. $\operatorname{Cos} \Theta_{0}$ as a function of $R_{0}^{-1}$ for dodecane, tetradecane, and hexadecane.

Here we have used that for a circular common line $\kappa_{\nu}^{(\mathrm{L}, \mathrm{S})}=$ $R_{0}^{-1}$. To use [43], we would need values for $\gamma_{(\mathrm{LS})}, \gamma_{(\mathrm{SV})}$, and $\sigma$. To our knowledge, there are no experimental data available for these parameters. This means that we cannot use Eq. [43] to calculate an a priori estimate for $\Theta_{0}$ that we could use for the solution of the inner problem.

Since we would still like to establish whether our method of solving the sessile drop problem is correct, we used the experimental data for the static contact angle $\Theta^{\text {(stat) }}$ from Gaydos and Neumann (13) as input. Using an iterative scheme, we calculated the value of $\Theta_{0}$ that would give us the experimental value for $\Theta^{\text {(stat) }}$. We did this for three systems, for several values of the radius of the sessile drop. In Fig. 2 we plot $\cos \Theta_{0}$ as a function of $R_{0}^{-1}$ for dodecane, tetradecane, and hexadecane. As predicted by [43], the results of the calculations all lie on a straight curve, which leads us to the conclude that our approach to solve the sessile drop problem is indeed correct. It also allows us to conclude that, as suggested by Gaydos and Neumann (13), line tension $\sigma$ is responsible for the dependance of the static contact angle on the radius of the sessile drop.
The slope of the curves in Fig. 2 is equal to $\sigma / \gamma_{(\mathrm{LV})}$, and the intersection with the $\cos \Theta_{0}$ axis is equal to $\left(\gamma_{(\mathrm{sv})}-\right.$ $\left.\gamma_{(\mathrm{LS})}\right) / \gamma_{(\mathrm{LV})}$. The values for these physical constants are given in Table 3 . The values for the line tensions are very close to those found by Gaydos and Neumann (13). However, their values for $\gamma_{(\mathrm{SV})}-\gamma_{(\mathrm{LS})}($ Table 3$)$ differ from ours (about $10 \%$ lower). The value for $\gamma_{(\mathrm{SV})}-\gamma_{(\mathrm{LS})}$ shows very little variation going from dodecane to hexadecane. For systems in which $\Theta_{0}$ is larger, the difference in the value for $\gamma_{(\mathrm{SV})}-\gamma_{(\mathrm{LS})}$ may be substantially more than $10 \%$.

There is an important difference between the work of Gaydos and Neumann (13) and that presented here. Unlike Gaydos and Neumann (13), we apply the momentum balance at the common line (generalized Young equation) at the true common line, with the intrinsic contact angle as the appropriate angle to be substituted in the equation. Gaydos and Neumann (13) follow a common practice of applying this equation to the outer region, with the static contact angle as the appropriate angle. In this approach, the momentum balance at the common line is interpreted as a force balance on a cylinder around the true common line. Although this approach is in principle correct, the equation must be corrected with a term that takes into account the differences in the bulk pressures on both sides of the liquid/vapor interface. As suggested by Benner et al. (40), the corrected momentum balance at the common line (for application to the outer region) should read

$$
\gamma_{(\mathrm{LS})}-\gamma_{(\mathrm{SV})}+\gamma_{(\mathrm{LV})} \cos \Theta^{(\mathrm{stat})}+\frac{\sigma}{R_{0}}+\Delta P=0
$$

In the analysis of their experimental data, Gaydos and Neumann (13) do not include the last term in [44].

\section{DETERMINATION OF SOLID - FLUID SURFACE TENSIONS}

The discussion in the preceding section allows us to formulate an improved scheme, to determine line tension and the difference between the fluid-solid surface tensions, using small sessile drops. The outline of this scheme is:

(i) Measure the static contact angle $\Theta^{\text {(stat) }}$ of the sessile

TABLE 3

Values for the Line Tension and for $\gamma_{(\mathrm{SV})}-\gamma_{(\mathrm{LS})}$ for Dodecane, Tetradecane, and Hexadecane

\begin{tabular}{lccc}
\hline Compound & $\sigma[\mathrm{J} / \mathrm{m}]$ & $\begin{array}{c}\gamma_{(\mathrm{SV})}-\gamma_{(\mathrm{LS})} \\
{\left[10^{-3} \mathrm{~N} / \mathrm{m}\right]}\end{array}$ & $\begin{array}{c}\gamma_{(\mathrm{SV})}-\gamma_{(\mathrm{LS})} \\
{\left[10^{-3} \mathrm{~N} / \mathrm{m}\right]^{a}}\end{array}$ \\
\hline Dodecane & $2.86 \times 10^{-6}$ & 21.7 & 19.6 \\
Tetradecane & $2.70 \times 10^{-6}$ & 21.9 & 19.4 \\
Hexadecane & $2.48 \times 10^{-6}$ & 21.6 & 19.1 \\
\hline
\end{tabular}

${ }^{a}$ Values for $\gamma_{(\mathrm{SV})}-\gamma_{(\mathrm{LS})}$ from Gaydos and Neumann (13). 
drop (using a microscope, with perhaps $10 \times$ magnification) as a function of the radius $R_{0}$ (using values for $R_{0}$ in the range of approximately $1-5 \mathrm{~mm}$ ).

(ii) Check if the inner [40] and outer [30] solutions are coupled, using the solution scheme outlined at the end of Section 4 ( setting $\Theta_{0}=0$ ).

(iii) If the inner and outer problem are not coupled, solve Eq. [40] consistent with [37] through [39], for the value of $\Theta_{0}$, using the experimental values of $\Theta^{\text {(stat) }}$ as input. This step gives $\Theta_{0}$ as a function of $R_{0}$.

(iv) Use Eq. [43] to determine the values of $\sigma$ and $\gamma_{(\mathrm{SV})}$ $-\gamma_{(\mathrm{LS})}$ from the $\left(\Theta_{0}, R_{0}\right)$ data points.

We expect this scheme to give more accurate values for $\gamma_{(\mathrm{SV})}-\gamma_{(\mathrm{LS})}$ than the conventional sessile drop method, in which a single large drop $\left(R_{0}>5 \mathrm{~mm}\right)$ is used.

\section{APPENDIX: NOMENCLATURE}

\section{Roman Symbols}

$a_{(i)}, b_{(i)}$ Coefficients in the emperical relation for the dielectric permeability

A Hamaker constant

$A^{*} \quad$ Dimensionless form of the Hamaker constant

$A_{0} \quad$ Unretarded limit of the Hamaker constant

$A_{0}^{*} \quad$ Dimensionless form of the unretarded limit of the Hamaker constant

$\mathbf{b}_{(i)} \quad$ Body force per unit mass of species $i$

$d \quad$ Minimum separation between liquid/vapor and liquid/solid interface

$d^{*} \quad$ Dimensionless form of $d$ in the outer solution

$d^{* *} \quad$ Dimensionless form of $d$ in the inner solution

$h^{\prime} \quad$ Film thickness

$h$

$h *$

$h * *$

$h_{0}$

$h_{0}^{*}$

$H$

$H^{*}$

$H^{* *}$

$n_{(i)}$

$N$

$N_{\text {B0 }}$

$P_{(i)}$

$r$

$r *$

$r_{(i j)}$

$R_{0}$ $s * *$

$T$

Dimensionless coordinate in the inner solution Temperature

\section{Greek Symbols}

$\gamma \quad$ Thermodynamic surface tension

$\Theta_{0} \quad$ Intrinsic contact angle

$\Theta^{\text {(stat) }} \quad$ Static contact angle

$\kappa_{\nu}^{(i, j)} \quad$ Geodesic curvature of the common line with respect to $\Sigma_{i, j}$

$\rho^{(\sigma)}$

$\rho^{(\mathrm{cl})}$

$\sigma$

$\phi_{(i j)}$

$\Phi_{(i)}$

$\Phi_{(i)}^{\mathrm{LvW}}$

Surface mass density

Line mass density

Thermodynamic line tension

pair potential per unit mass of $i$ and unit mass of j

Potential energy per unit mass of $i$

London-van der Waals contribution to $\Phi_{(i)}$

\section{REFERENCES}

1. Sagis, L. M. C., and Slattery, J. C., J. Colloid Interface Sci. 176, 150 (1995).

2. Sagis, L. M. C., and Slattery, J. C., J. Colloid Interface Sci. 176, 165 (1995).

3. de Feijter, J. A., and Vrij, A., J. Electroanal. Chem. 37, 9 (1972).

4. Platikanov, D., Nedyalkov, M., and Scheludko, A., J. Colloid Interface Sci. 75, 612 (1980).

5. Platikanov, D., Nedyalkov, M., and Nasteva, V., J. Colloid Interface Sci. 75, 620 (1980).

6. Kralchevsky, P. A., and Ivanov, I. B., Chem. Phys. Lett. 121, 116 (1985).

7. Kralchevsky, P. A., and Ivanov, I. B., J. Colloid Interface Sci. 137, 234 (1990).

8. Langmuir, I., J. Chem. Phys. 1, 756 (1933).

9. Harkins, W. D., J. Chem. Phys. 5, 135 (1937).

10. Torza, S., and Mason, S. G., Kolloid Z. Z. Polym. 246, 593 (1971).

11. Good, R. J., and Koo, M. N., J. Colloid Interface Sci. 71, 283 (1979).

12. Starov, V. M., and Churaev, N. V., Kolloidn Zh. 42, 703 (1980).

13. Gaydos, J., and Neumann, A. W., J. Colloid Interface Sci. 120, 76 (1987).

14. Wallace, J. A., and Schurch, S., J. Colloid Interface Sci. 124, 452 (1988).

15. Yekta-Fard, M., and Ponter, A. B., J. Colloid Interface Sci. 126, 134 (1988).

16. Li, D., Lin, F. Y. H., and Neumann, A. W., J. Colloid Interface Sci. 142, 224 (1991).

17. Drelich, J., Miller, J. D., and Hupka, J., J. Colloid Interface Sci. 155, 379 (1993).

18. Grets, R. D., Surf. Sci. 5, 249 (1982).

19. Navascues, G., and Mederos, L., Surf. Technol. 17, 79 (1982).

20. Lazaridis, M., J. Colloid Interface Sci. 155, 386 (1993).

21. Churaev, N. V., and Starov, V. M., J. Colloid Interface Sci. 103, 301 (1985).

22. Bashforth, F., and Adams, J. C., "An Attempt to Test the Theories of Capillary Action.' Cambridge Univ. Press, Cambridge, 1883.

23. Blaisdell, B. E., J. Math. Phys. 19, 186 (1940).

24. Blaisdell, B. E., J. Math. Phys. 19, 217 (1940).

25. Blaisdell, B. E., J. Math. Phys. 19, 228 (1940).

26. Staicopolus, D. N., J. Colloid Sci. 17, 439 (1962).

27. Staicopolus, D. N., J. Colloid Sci. 18, 793 (1963).

28. Staicopolus, D. N., J. Colloid Sci. 23, 453 (1967). 
29. Padday, J. F., Phil. Trans. R. Soc. London A 269, 265 (1971).

30. Padday, J. F., and Pitt, A., J. Colloid Interface Sci. 38, 323 (1972).

31. Hartland, S., and Hartley, R. W., "Axisymmetric Fluid-Liquid Interfaces," Elsevier, Amsterdam, 1976.

32. Dzyaloshinskii, I. E., Lifshitz, E. M., and Pitaevskii, L. P., Sov. Phys. JETP Engl. Transl. 37, 161 (1960).

33. Padday, J. F., and Uffindell, N. D., J. Phys. Chem. 72, 1407 (1968).

34. Hough, D. B., and White, L. R., Adv. Colloid Interface Sci. 14, 3 (1980).

35. Young, T., Philos. Trans. R. Soc. London 95, 65 (1805).

36. Israelachvili, J. N., J. Chem. Soc. Faraday Trans. 2 69, 1729 (1973).

37. Miller, C. A., and Ruckenstein, E., J. Colloid Interface Sci. 48, 368 (1974).

38. Jameson, G. J., and del Cerro, M. C. G., J. Chem. Soc. Faraday Trans. 1 72, 883 (1976).

39. Saville, G., J. Chem. Soc. Faraday Trans. 2 73, 1122 (1977).

40. Benner, R. E., Scriven, L. E., and Davis, H. T., Faraday Symp. Chem. Soc. 16, 169 (1981).

41. Wayner Jr., P. C., J. Colloid Interface Sci. 77, 495 (1980).

42. Wayner Jr., P. C., J. Colloid Interface Sci. 88, 294 (1982).

43. Slattery, J. C., 'Interfacial Transport Phenomena.' Springer-Verlag, Berlin, 1990.

44. Fox, H. W., and Zisman, W. A., J. Colloid Sci. 5, 514 (1950).

45. Christenson, H. K., Gruen, D. W. R., Horn, R. G., and Israelachvili, J. N., J. Chem. Phys. 87, 1834 (1987).

46. Israelachvili, J. N., Acc. Chem. Res. 20, 415 (1987).
47. Israelachvili, J. N., "Intermolecular and Surface Forces." 2nd ed. Academic Press, San Diego, 1991.

48. Israelachvili, J. N., and McGuiggan, P. M., Science 241, 795 (1988).

49. Homola, A. M., Israelachvili, J. N., Gee, M. L., and McGuiggan, P. M., J. Tribol. 111, 675 (1989).

50. Hirschfelder, J. O., Curtiss, C. F., and Bird, R. B., " Molecular Theory of Gases and Liquids." Wiley, New York, 1954.

51. Maitland, G. C., Rigby, M., Smith, E. B., and Wakeham, W. A., "'Intermolecular Forces.', Clarendon, Oxford, 1981.

52. Casimir, H. B. G., and Polder, D., Phys. Rev. 73, 360 (1948).

53. Hamaker, H. C., Physica IV 10, 1058 (1937).

54. Lifshitz, E. M., Sov. Phys. JETP Engl. Transl. 2, 77 (1956).

55. Dzyaloshinskiî, I. E., Lifshitz, E. M., and Pitaevskiǐ, L. P., Adv. Phys. 10, 165 (1961).

56. Scheludko, A., Platikanov, D., and Manev, E., Disc. Faraday Soc. 40, 253 (1965).

57. Ruckenstein, E., and Jain, R. K., J. Chem. Soc. Faraday Trans. 2 70, 132 (1974).

58. Vassilieff, C. S., and Ivanov, I. B., Colloid Polym. Sci. 254, 431 (1976).

59. Vassilieff, C. S., and Ivanov, I. B., Z. Naturforsch. A: Phys. Phys. Chem. Kosmophys. 31, 1584 (1976).

60. Vassilieff, C. S., and Ivanov, I. B., C. R. Acad. Sci. Bulg. 29, 1795 (1976).

61. Nir, S., and Vassilieff, C. S., in "Thin Liquid Films"' (I. B. Ivanov, Ed.), Vol. 29 p. 207. Dekker, New York, 1988.

62. Krupp, H., Adv. Colloid Interface Sci. 1, 111 (1967). 